\title{
Absolute spectroscopic factors from neutron knockout on the halo nucleus ${ }^{15} \mathrm{C}$
}

\author{
J. R. Terry, ${ }^{1,2}$ D. Bazin, ${ }^{1}$ B. A. Brown, ${ }^{1,2}$ J. Enders, ${ }^{1, *}$ T. Glasmacher, ${ }^{1,2}$ \\ P. G. Hansen, ${ }^{1,2, \dagger}$ B. M. Sherrill, ${ }^{1,2}$ and J. A. Tostevin ${ }^{3}$ \\ ${ }^{1}$ National Superconducting Cyclotron Laboratory, Michigan State University, East Lansing, Michigan 48824, USA \\ ${ }^{2}$ Department of Physics and Astronomy, Michigan State University, East Lansing, Michigan 48824, USA \\ ${ }^{3}$ Department of Physics, School of Electronics and Physical Sciences, University of Surrey, \\ Guildford, Surrey GU2 7XH, United Kingdom
}

(Received 15 January 2004; published 14 May 2004)

\begin{abstract}
An accurate determination of the partial cross sections to four final states in the ${ }^{9} \mathrm{Be}\left({ }^{15} \mathrm{C},{ }^{14} \mathrm{C}\right) X$ reaction has been carried out at $103 \mathrm{MeV} /$ nucleon. The cross section of $101(5) \mathrm{mb}$ to the ${ }^{14} \mathrm{C} 0^{+}$ground state is of special interest. Relative to the theoretical cross section calculated on the basis of the spectroscopic factor from effective-interaction theory, this amounts to a quenching factor $R_{s}=0.90(4)(5)$. Here the first number in parentheses is the experimental error, and the second is the error on the theoretical unit (reaction) cross section. The result gives support to the idea that weakly bound halolike states have quenching factors that approach unity, in contrast to factors of $0.5-0.6$ characteristic of well-bound states in nuclei near stability.
\end{abstract}

DOI: 10.1103/PhysRevC.69.054306 PACS number(s): 21.10.Jx, 21.60.Cs, 24.50.+g, 27.20.+n

\section{INTRODUCTION}

Many-body theory based on the independent-particle shell model has enjoyed great success in interpreting nuclear structure. As usually applied, it makes the problem numerically tractable by introducing truncated orbital spaces. Inside these, the physical interactions are replaced by effective interactions, tailored to reproduce experimental data, such as masses and excitation energies. Imposing these restrictions excludes probability from states at higher energies, thereby inflating the populations within the truncated model space. The wave functions now describe quasiparticles, and matrix elements and occupancies may differ systematically from the observed values [1]. In the present work, the absolute occupancy of the $1 s_{1 / 2}$ neutron halo state of ${ }^{15} \mathrm{C}$ is investigated through a precise measurement of spectroscopic factors in the reaction ${ }^{9} \mathrm{Be}\left({ }^{15} \mathrm{C},{ }^{14} \mathrm{C}\right) X$. This is an especially favorable case in which the contributions from configuration mixing are well understood and expected to be small.

\section{ABSOLUTE SPECTROSCOPIC FACTORS OF WEAKLY BOUND SYSTEMS}

Experiments on stable nuclei, mainly for cases involving closed shells, have shown that strength is missing relative to the predictions of effective-interaction theory. Although a quantitative description is not yet available, this effect is believed to be caused by components of the nucleon-nucleon interaction that are absent in the usual nuclear models, such as the repulsive hard core and the tensor force terms [2]. Other effects not included in central-field models such as clustering could also contribute to this effect. We denote the empirical reduction factor by the symbol $R_{s}$, which is, of

\footnotetext{
*Present address: Institut für Kernphysik, Technische Universität, Darmstadt, Germany.

${ }^{\dagger}$ Electronic address: hansen@nscl.msu.edu
}

course, a model-dependent quantity. An illustrative first example has been provided by the ${ }^{7} \operatorname{Li}\left(e, e^{\prime} p\right){ }^{6} \mathrm{He}$ reaction, for which the sum of the measured spectroscopic factors to the two lowest states is $0.58(5)$, in excellent agreement with the value 0.60 obtained in a variational Monte Carlo calculation that starts from realistic nucleon-nucleon forces [3]. The Cohen-Kurath spectroscopic factor based on a $p$-shell space and including a center-of-mass correction of 7/6 gives essentially twice this value, 1.16, so that here we have $R_{s}$ $=0.50$. The proton knockout reaction with high-energy electron beams has been employed over the past two decades to study absolute spectroscopic factors for proton states in stable nuclei over a broad mass region [4], and has consistently found similar values for $R_{s}$, of the order of $0.5-0.6$ relative to shell-model and mean-field models that use effective interactions.

It is only recently that evidence has emerged $[5,6]$ that nucleon knockout reactions with heavy ions, at intermediate energies and in inverse kinematics, offer new possibilities for studying absolute spectroscopic factors. The strength of this approach lies in the accurate understanding of the (unit) single-particle cross sections $\sigma_{\text {sp }}$ (i.e., corresponding to a spectroscopic factor of 1), which serve to convert measured partial cross sections to experimental spectroscopic factors. The calculations of $\sigma_{\mathrm{sp}}$ rely on the high-energy limit of reaction theory, based on eikonal methods. These offer formal, practical, and quantitative advantages over conventional transfer-reaction approaches [7-9]. The proton states of the stable $p$-shell nuclei ${ }^{12} \mathrm{C}$ and ${ }^{16} \mathrm{O}$ were found by this method to have $R_{s}$ values of $0.57(2)$ and $0.68(4)$ [5] in good agreement with what had been obtained from the $\left(e, e^{\prime} p\right)$ reaction. However, the method is equally applicable to neutron states; the values for the same two nuclei were $0.52(2)$ and $0.56(3)$, respectively. This is a new result but, of course, what should be expected from isospin symmetry. The results for $R_{s}$ for the two radioactive nuclei, ${ }^{8} \mathrm{~B}$ and ${ }^{9} \mathrm{C}$, were considerably higher, $0.872(12)$ (weighted average over five measured values) and $0.82(6)$, respectively $[5,6]$. The values closer to unity are, 
most likely, associated with the low proton binding for the two nuclei, 0.14 and $1.3 \mathrm{MeV}$, respectively. If so, the effect would not have been observable in $\left(e, e^{\prime} p\right)$ work, which could only investigate more bound stable targets. It is clearly of interest to investigate other weakly bound cases. However, although many partial cross sections have been investigated in knockout reactions [9-11], the majority do not fulfill the stringent experimental and theoretical requirements for a precise determination of $R_{s}$.

The single-neutron knockout on the nucleus ${ }^{15} \mathrm{C}\left(\frac{1}{2}{ }^{+}\right)$leading to the $0^{+}$ground state of ${ }^{14} \mathrm{C}$ presents an almost ideal case for a precision test. The initial state is halolike with a neutron separation energy of only $1.218 \mathrm{MeV}$. Shell-model calculations indicate very weak configuration mixing for this state. The $p$-sd interaction set given by Warburton and Brown [12], referred to as WBT, gives a spectroscopic factor of 0.983 , very close to the sum-rule value of unity for a pure $1 s_{1 / 2}$ state. Other sets give very similar values. Therefore, the sensitivity to the structure model should be small. The reaction is also very favorable from an experimental point of view. The reaction has been studied at $54 \mathrm{MeV} /$ nucleon and the spectroscopic factors were discussed [13]. The most interesting result was a deviation in the shape of the knockout momentum distribution, which was attributed to non-eikonal effects in the diffractive-breakup channel. However, in spite of this, the integrated single-particle cross section could be accounted for in eikonal theory. From this work it is also known that about $25 \%$ of the reaction populates excited states in ${ }^{14} \mathrm{C}$. Determining the intensity of these branches with a $\pm 10 \%$ error will then determine the branching ratio to the ground state to a comfortable error of $\pm 2.5 \%$. This branching ratio combines with an accurately determined inclusive $\left({ }^{15} \mathrm{C},{ }^{14} \mathrm{C}\right)$ cross section to give the partial cross section of interest.

It was decided to perform the precise determination of the partial cross section at an incident energy of $103 \mathrm{MeV} /$ nucleon. Using a higher energy than in the experiment of Ref. [13] brings experimental advantages, which include the possibility of using a thicker target and, very importantly, a marked improvement in the acceptance of the spectrograph, both with respect to angle and transmitted momentum bite. On the theoretical side, the analysis becomes safer because the precision of eikonal reaction theory improves with increasing projectile energy, but this no longer seems an important issue. We note in this connection that five separate experiments on ${ }^{8} \mathrm{~B}$ obtained consistent spectrocopic factors within a range of beam energies from 0.076 to 1.4 MeV/nucleon [6].

\section{EXPERIMENT AND DATA ANALYSIS}

Two separate experiments were carried out at the National Superconducting Cyclotron Laboratory (NSCL) at MSU. In the first, a secondary beam of $103 \mathrm{MeV} /$ nucleon ${ }^{15} \mathrm{C}$ was produced by fragmentation of a $150 \mathrm{MeV} /$ nucleon ${ }^{17} \mathrm{O}$ primary beam on a thick beryllium target and subsequently purified by magnetic analysis in the A1200 fragment separator [14]. This beam was transported to the target position of the S800 spectrograph [15], where it interacted with a

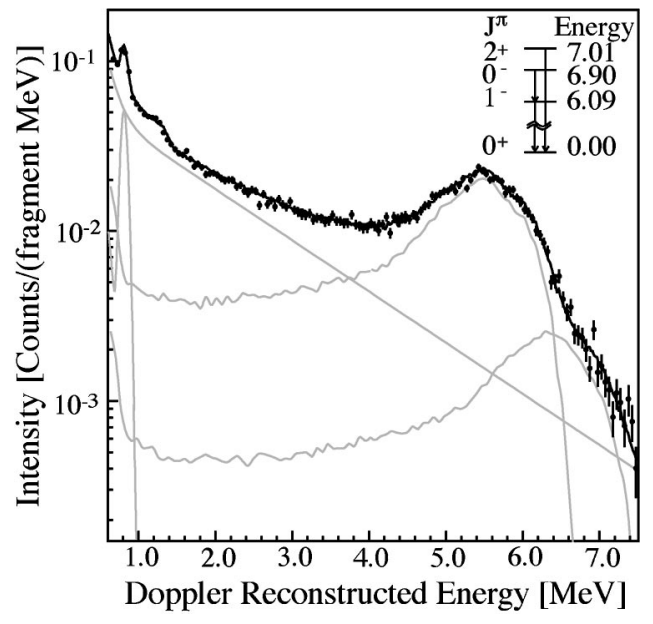

FIG. 1. The $\gamma$-ray spectrum from the reaction ${ }^{9} \mathrm{Be}\left({ }^{15} \mathrm{C},{ }^{14} \mathrm{C}\right.$ $+\gamma) X$ (filled circles) transformed, event by event, to the projectile frame at midtarget. The response functions for each gamma energy arising from this analysis have been obtained in the Monte Carlo simulation. The approximately exponential component is the continuum contribution ascribed to neutrons and gamma rays from the target. Its intensity and shape has been taken from [17]. The inset shows the simplified level scheme of ${ }^{14} \mathrm{C}$ indicating the states populated in this reaction.

$149 \mathrm{mg} / \mathrm{cm}^{2}$ beryllium target. The residues were analyzed in the spectrograph in coincidence with prompt gamma emissions detected in an array of 11 position-sensitive $\mathrm{NaI}$ detectors [16]. The spectrograph was operated in dispersion matched mode for maximum momentum resolution at the focal plane. A detailed discussion of techniques and analysis in this part of the experiment has been given in connection with a study of other radioactive carbon isotopes [17]. The inclusive cross section, obtained from the number of detected residues relative to the number of incident ${ }^{15} \mathrm{C}$ projectiles, was $137.0(71) \mathrm{mb}$ without acceptance corrections. These are discussed below.

The coincident gamma-ray spectrum, shown in Fig. 1, was fit GEANT-simulated response functions, which account for the efficiency of the detector array and distortions in the response function due to the Doppler effect. Gamma ray energies for this calculation are taken from previous work [27]. To quantify the accuracy of the simulation, a calibration run with stationary radioactive sources was carried out; for this, the simulated data agree in intensity with the actual data at an uncertainty level of $10 \%$. The fit to the gamma spectrum also included a previously observed continuum distribution. The intensity and shape of this distribution are consistent with measurements [17] of single-neutron removal from ${ }^{12} \mathrm{Be}$ and ${ }^{16} \mathrm{C}$, for which there are no gammas above $1 \mathrm{MeV}$. Further, the fit includes a small contribution from a $0.98 \mathrm{MeV}$ stationary-source gamma ray associated with a ${ }^{8} \mathrm{Li}$ contamination in the target. The fit shown in the figure leads to apparent absolute (not acceptance corrected) gamma intensities of $4.32(50) \%, 22.4(23) \%$, and $3.4(10) \%$ for the 0.809 , 6.092 , and $7.010 \mathrm{MeV}$ peaks, respectively. (The intensities are reported relative to the number of observed residues). The input-output intensity balance leads to apparent population branches (again not corrected for acceptance) of 
TABLE I. Results for the reaction ${ }^{9} \mathrm{Be}\left({ }^{15} \mathrm{C},{ }^{14} \mathrm{C}\left(I^{\pi}\right)+\gamma\right) X$ at $E=103 \mathrm{MeV} /$ nucleon. The table lists the orbital angular momenta $\ell$, the theoretical spectroscopic factors $S$, the single-particle cross sections (in $\mathrm{mb}$ ) for the stripping and diffraction dissociation mechanisms, as well as the resulting theoretical partial cross sections $\sigma_{\text {th }}$. The experimental branching ratios to individual final levels, combined with the measured total (inclusive) cross section as discussed in the text, lead to the experimental partial cross sections.

\begin{tabular}{llllrrrrc}
\hline \hline$E(\mathrm{MeV})$ & $I^{\pi}$ & $\ell$ & $S_{\text {th }}$ & $\sigma_{\text {sp }}^{\text {str }}$ & \multicolumn{1}{c}{$\sigma_{\text {sp }}^{\text {dif }}$} & \multicolumn{1}{c}{$\sigma_{\text {th }}$} & \multicolumn{1}{c}{$b_{\exp }$} & \multicolumn{1}{c}{$\sigma_{\exp }$} \\
\hline 0.000 & $0^{+}$ & 0 & 0.983 & 62.1 & $37.3^{\mathrm{a}}$ & 112.2 & $0.718(24)$ & $100.8(44)$ \\
6.094 & $1^{-}$ & 1 & 1.197 & 22.6 & $6.4^{\mathrm{b}}$ & 37.2 & $0.196(24)$ & $27.4(41)$ \\
6.903 & $0^{-}$ & 1 & 0.457 & 21.7 & $5.9^{\mathrm{b}}$ & 13.5 & $0.046(6)$ & $6.5(9)$ \\
7.012 & $2^{+}$ & 2 & 0.016 & 22.8 & $5.9^{\mathrm{b}}$ & 0.5 & $0.039(12)$ & $5.5(17)$ \\
\hline & & & & & & & $\sigma_{\text {tot }}$ & $140.2(46)$ \\
\hline
\end{tabular}

${ }^{\mathrm{a}}$ This diffraction-dissociation cross section to the ground state includes a calculated Coulomb contribution of $2.9 \mathrm{mb}$.

${ }^{\mathrm{b}}$ The single-particle cross sections to these final states were calculated assuming a ${ }^{13} \mathrm{C}+n(1 s)$ composite core [7].

$74.2(25) \%, 18.1(23) \%, 4.32(50) \%$, and $3.4(10) \%$ for the $0^{+}$, $1^{-}, 0^{-}$, and $2^{+}$states, respectively.

During the measurement of the inclusive ${ }^{9} \mathrm{Be}\left({ }^{15} \mathrm{C},{ }^{14} \mathrm{C}\right) \mathrm{X}$ cross section, the incident beam and the reaction residues are observed at different field settings and, therefore, different times. The rate and composition must be carefully monitored to safeguard against drift. Since the measurement of the inclusive ${ }^{9} \mathrm{Be}\left({ }^{15} \mathrm{C},{ }^{14} \mathrm{C}\right) \mathrm{X}$ cross section represents the most delicate part of the present high-precision study, it was decided to repeat this at the NSCL's Coupled-Cyclotron Facility. As the new setup employed a high-purity germanium array for gamma detection with a relatively low efficiency at $6 \mathrm{MeV}$ as compared to the $\mathrm{NaI}$ array used in the first experiment, and since the run was short, there were not enough statistics to allow a remeasurement of the branching ratios. A secondary beam of ${ }^{15} \mathrm{C}$ at $103 \mathrm{MeV} /$ nucleon was produced by fragmentation of ${ }^{40} \mathrm{Ar}$ and purified in the A1900 fragment separator [18]. The secondary beam interacted with a beryllium target with nominal thickness of $376 \mathrm{mg} / \mathrm{cm}^{2}$ located at the target position of the S800. The target thickness was determined independently by measuring the magnetic fields required to bring a ${ }^{30} \mathrm{Si}$ beam to the final focus of the $\mathrm{S} 800$ spectrograph with and without the target. From the energy loss, a target thickness of $375(4) \mathrm{mg} / \mathrm{cm}^{2}$ was found. The S800 was operated in focused mode, which gives a higher event rate and a more sharply defined beam spot on the target. Particle identification was achieved by selection on energy loss versus time of flight to the focal plane of the spectrograph. The time of flight was corrected for trajectory dependence yielding a very clean identification of the ${ }^{14} \mathrm{C}$ events with an error of $2.3 \%$ attributed to the selection of the residues. Other contributions to the error on the inclusive cross section are $1.5 \%$ from the normalization to the incident beam, $1 \%$ from the target thickness, $0.6 \%$ from the live time of the data recording, and $0.9 \%$ from counting statistics. The resulting apparent inclusive cross section for single-neutron removal was $133.0(41) \mathrm{mb}$, in agreement with the value $137.0(71) \mathrm{mb}$ obtained in the first measurement. We represent the two independent results by a weighted average of 134.0(36) mb, and now introduce the corrections for mo- mentum acceptance. Since the shapes of the momentum distributions are $\ell$-dependent (high $\ell$ values have broader distributions), this required use of the coincidence data.

Acceptance corrections for detection of reaction residues were determined in a Monte Carlo calculation employing the COSY Infinity code for tracking charged particles through the S800 spectrograph. Because of the relatively high energy in this experiment, the angular acceptance is essentially complete, and the corrections depend on the longitudinal momentum distributions in coincidence with gamma rays measured (and calculated) as in [13] with the ground-state momentum distribution being obtained as a difference. The contribution from the continuum distribution in Fig. 1 was extracted by gating in the $E_{\gamma}$ region between the 0.809 and $6.092 \mathrm{MeV}$ peaks, where the continuum distribution dominates the spectrum. All the momentum distributions were weighted with factors extracted from the gamma fit. Statistics in the $7.010 \mathrm{MeV}$ peak, associated with the $2^{+}$state, were too small to yield a momentum distribution, but we assume the theoretical $\ell=2$ shape. (For measured shapes from $d$-wave removal in the same mass range, see [17].) The resulting acceptance corrections are $1.4(10) \%$ for the $0^{+}$state ( $s$-wave removal), $13(8) \%$ for the $0^{-} / 1^{-}$states ( $p$-wave removal), and $20(10) \%$ for the $2^{+}$state. Applying the corrections to the measured relative cross sections leads to an acceptance correction factor of 1.046(20) to the inclusive cross section, which becomes 140.2(46) mb at $103 \mathrm{MeV} /$ nucleon. The corresponding partial cross sections and branching ratios are given in Table I. We have used the experimental spectroscopic factors defined by the results of Table I for calculating the cross sections and branching ratios that would have been obtained in the experiment at $54 \mathrm{MeV} /$ nucleon [13]. The agreement turns out to be very good for the branching ratios. However, the calculated inclusive cross section comes out as 163(5) mb, less than two standard deviations above the reported $54-\mathrm{MeV}$ value of $137(16)$. We take this as an indication of a possible systematic error. The new measurement is clearly more accurate and should supersede that at $54 \mathrm{MeV} /$ nucleon. 


\section{THEORETICAL ANALYSIS}

The calculation of theoretical spectroscopic reference cross sections combine shell-model theory based on effective interactions with eikonal reaction theory. For a given final level identified by $I^{\pi}$, the cross section can be be written

$$
\sigma_{\mathrm{th}}\left(I^{\pi}\right)=\left(\frac{A}{A-1}\right)^{N} S \sigma_{\mathrm{sp}}\left(\ell, B_{n}\right),
$$

where the $A$-dependent term is a center-of-mass correction [19], $N$ is the oscillator quantum number for the orbital in question, $S$ is the spectroscopic factor, and $\sigma_{\mathrm{sp}}\left(\ell, B_{n}\right)$ is the (unit) single-particle cross section. Since the latter in our analysis depends essentially on the orbital angular momentum $\ell$ and the neutron binding energy $B_{n}$, we have, for the $1^{-}$and $2^{+}$states in Table I, combined the two $j$ components to give a single spectroscopic factor $S$. (The dominant components in the two cases are $88 \% 0 p_{1 / 2}$, and $89 \% 0 d_{5 / 2}$, respectively.) As described in [7-9], the single-particle cross sections were calculated in a threebody eikonal model based on the approximation that the $(A-1)$-body residue acts as a spectator, so that it is at most scattered elastically by the target. The nucleon-residue relative motion is calculated as an energy eigenstate of an effective two-body Hamiltonian containing a WoodsSaxon potential with a depth adjusted to reproduce the sum of the nucleon separation energy and the excitation energy of the final state. The residue-core and nucleoncore interactions are expressed in terms of elastic $S$ matrices.

The elastic $S$ matrices for the reactions of the nucleon and the core (reaction residue) were obtained from the eikonal model by single and double folding, respectively, of the nucleon-nucleon interaction. The nuclear matter distributions were assumed to be Gaussian. For the excited states in ${ }^{14} \mathrm{C}$, the core $S$ matrix was calculated with the composite-core prescription [7] to correct for the presence of the halo neutron as a spectator in the reaction residue. Based on a ${ }^{13} \mathrm{C}$ radius of $2.29 \mathrm{fm}$, this reduces the single-particle cross section for the $1^{-}$level from $30.9 \mathrm{mb}$ to the value of $29.0 \mathrm{mb}$ given in Table I. For the ground state, the values $R_{C}$ $=2.36(5)$ and $R_{T}=2.37(1)$ for the matter radii of the core and of the target, respectively, were taken as the average of (consistent) values obtained from the charge radii [25] corrected for the proton charge radius of $0.85 \mathrm{fm}$, and from the interaction cross sections [26].

The simple and accurate description of the knockout reaction is linked to the surface dominance of the reaction mechanism. The fraction of the neutron wave function sampled in the reaction can be estimated to be $28 \%$, obtained as the ratio of the stripping cross section $(6.21 \mathrm{mb}$, discussed below) to the total reaction cross section for a free neutron, $228 \mathrm{mb}$. As a result of this surface dominance, treatments that use a different description of the nuclear interior give very similar overall results [8]. To allow for a general comparison of results from single-nucleon removal studies, a standard for the core-nucleon interaction is required. The early, less precise spectroscopic work, see [10], characterized the shape of the core-nucleon potential well with a fixed set of Woods-Saxon parameters, referred to as the "standard set" [5]. However, the recent work on absolute spectroscopic factors $[5,6]$, requiring an improvement in precision, has relied on electromagnetic properties such as higher moments of the charge radii and Coulomb displacement energies for fixing these parameters in special cases. As such data are not available for a large number of nuclides, we shall argue here that a more general approach for precision studies is to use selfconsistent Hartree-Fock calculations to fix the Woods-Saxon parameters.

Before we discuss Hartree-Fock calculations, it is important to consider the three parameters that we wish to fix. The depth of the nucleon-residue potential well is an important parameter in surface-dominated processes due to its influence on the asymptotic behavior of the wave function. This parameter is fixed precisely by the binding energy of the single-particle orbit, which is the sum of the neutron separation energy and excitation energy of the final state (as described above). The last two parameters describe the extent and shape of the distribution, typically defined as a radius and a diffuseness. However, a numerical study of the sensitivity of single-particle cross sections to these parameters by means of finite-difference derivatives has revealed that more natural coordinates for this calculation are the rms radius of the orbit and the diffuseness of the potential well [9]. Calculated single-particle cross sections become insensitive to the diffuseness of the potential once the rms radius is fixed. This is illustrated in our final calculations below. In these calculations we fix the diffuseness at a reasonable value of $0.5 \mathrm{fm}$.

Modern Hartree-Fock (HF) calculations based on Skyrme parametrizations provide a quantitative agreement with experiment for a number of parameters related to nuclear size. We use here the recent parameter set SKX [20], which has been determined from a large set of data on spherical nuclei, including nuclei far from stability. With this it has been possible to account for the binding-energy differences between mirror nuclei [21], interaction cross sections [22], and the nuclear charge distributions [23]. The good agreement with a large body of data suggests that the theory will also give a good description of the radial distribution of individual orbits. Due to the strong sensitivity of the single-particle cross section to the binding energy, if is necessary in the HF calculation to scale the central potential by a number near unity to reproduce this quantity for the orbital in question. Since the $\mathrm{HF}$ radius is defined with a fixed center of the nucleus, a conversion is required to find the radius of the single particle orbit in core-nucleon coordinates. This relation is given by

$$
R_{\mathrm{sp}}^{2}=\left\langle r^{2}\right\rangle=\left(\frac{A}{A-1}\right)\left\langle r_{\mathrm{HF}}^{2}\right\rangle .
$$

The calculated Hartree-Fock radius for the $1 s_{1 / 2}$ state of ${ }^{15} \mathrm{C}$ is $5.01 \mathrm{fm}$, which translates into a two-body rms distance of $5.185 \mathrm{fm}$. With the diffuseness fixed at $a=0.5 \mathrm{fm}$, the rms is reproduced by the Woods-Saxon parameter $r_{0}=1.223 \mathrm{fm}$. With this choice we obtain for a $103 \mathrm{MeV} /$ nucleon ${ }^{15} \mathrm{C}$ projectile on a ${ }^{9} \mathrm{Be}$ target a contribution from stripping of $62.1 \mathrm{mb}$ and from diffraction dissociation $34.5 \mathrm{mb}$. The latter result is based on a sum rule which assumes no bound 
excited states [28]. This should be an excellent approximation for ${ }^{15} \mathrm{C}$ since it has only one bound excited state. To this cross section we must add a small contribution from Coulomb dissociation, calculated to be $2.9 \mathrm{mb}$ [24], leading to the (unit) single-particle cross section of $99.4 \mathrm{mb}$ given in Table I. The cross sections for the $1^{-}$state are based on an $R_{\mathrm{sp}}$ of $3.167 \mathrm{fm}$ yielding the Woods-Saxon parameters $(1.402,0.5)$, which were also used for the two small cross sections. As a check on the procedure, we calculated the $1 s_{1 / 2}$ cross section with the Woods-Saxon diffuseness increased to $a=0.55$. The same value for $R_{\mathrm{sp}}$ is obtained by choosing $r_{0}$ $=1.113 \mathrm{fm}$, which leads to stripping and diffraction cross sections of 62.1 and $34.3 \mathrm{mb}$, a change of $-0.2 \%$.

The precision in the calculation of the theoretical unit cross sections (given as the sum of the contributions from stripping and diffraction dissociation) may be estimated from finite-difference derivatives

$$
\frac{\delta \sigma_{\mathrm{sp}}}{\sigma_{\mathrm{sp}}}=0.44 \delta R_{\mathrm{sp}}-0.04 \delta a-0.31 \delta R_{C}-0.31 \delta R_{T},
$$

with the coefficients in $\mathrm{fm}^{-1}$. This shows that the cross section is insensitive to the choice of the diffuseness parameter $a$. With estimated errors on the single-particle rms radius and the diffuseness of $0.1 \mathrm{fm}$, and with errors on the radii of $0.05 \mathrm{fm}$, the resulting mean-square error on the unit cross section is $5 \%$, comparable to the experimental error. For the more deeply bound $1^{-}$state, the relative error is $8.5 \%$, mainly associated with the radius of the single-particle orbital (coefficient $0.85 \mathrm{fm}^{-1}$ ), while the coefficients describing the dependence on the nuclear radii are only $0.02 \mathrm{fm}^{-1}$.

\section{CONCLUSION}

In order to compare the experimental result for the $1 s_{1 / 2}$ knockout to the ground state with effective-interaction theory, we introduce the quenching factor defined in $[5,6]$,

$$
R_{s}=\frac{\sigma_{\text {exp }}}{\sigma_{\text {th }}}=0.90(4)(5),
$$

where the first quantity in parentheses is the experimental error (standard deviation) and the second represents our estimate of the uncertainty on the unit cross section. The result is similar to those for ${ }^{8} \mathrm{~B}$ and ${ }^{9} \mathrm{C}$ discussed in Sec. II and confirms that halolike states have quenching factors approaching unity. This is what one would intuitively expect for a neutron in a very large $s$-state halo. As such states extend far beyond the core of the nucleus, their behavior must approach that of a free particle.

\section{ACKNOWLEDGMENTS}

This work was supported by the U.S. National Science Foundation under Grant Nos. PHY-01 10253 and PHY-00 70911 and by the United Kingdom Engineering and Physical Sciences Research Council (EPSRC) Grant No. GR/ M82141. The authors would like to thank the NSCL accelerator and beam delivery staff as well as the collaborators from experiments NSCL 98006 and 01013.
[1] B. A. Brown, Prog. Part. Nucl. Phys. 47, 517 (2001).

[2] V. R. Pandharipande, I. Sick, and P. K. A. de Witt-Huberts, Rev. Mod. Phys. 69, 981 (1997).

[3] L. Lapikas, J. Wesseling, and R. B. Wiringa, Phys. Rev. Lett. 82, 4404 (1999).

[4] G. J. Kramer, H. P. Blok, and L. Lapikas, Nucl. Phys. A679, 267 (2001).

[5] B. A. Brown, P. G. Hansen, B. M. Sherrill, and J. A. Tostevin, Phys. Rev. C 65, 061601(R) (2002).

[6] J. Enders et al., Phys. Rev. C 67, 064301 (2003).

[7] J. A. Tostevin, J. Phys. G 25, 735 (1999).

[8] J. A. Tostevin, Nucl. Phys. A682, 320c (2001).

[9] P. G. Hansen and J. A. Tostevin, Annu. Rev. Nucl. Part. Sci. 53, 219 (2003).

[10] P. G. Hansen and B. M. Sherrill, Nucl. Phys. A693, 133 (2001).

[11] E. Sauvan et al., Phys. Lett. B 491, 1 (2000).

[12] E. K. Warburton and B. A. Brown, Phys. Rev. C 46, 923 (1992).

[13] J. A. Tostevin et al., Phys. Rev. C 66, 024607 (2002)

[14] B. M. Sherrill et al., Nucl. Instrum. Methods Phys. Res. B 70, 298 (1992).

[15] D. Bazin et al., Nucl. Instrum. Methods Phys. Res. B 204, 629
(2003)

[16] H. Scheit et al., Nucl. Instrum. Methods Phys. Res. A 422, 124 (1999).

[17] V. Maddalena et al., Phys. Rev. C 63, 024613 (2001).

[18] D. J. Morrissey et al., Nucl. Instrum. Methods Phys. Res. B 203, 90 (2003).

[19] A. E. L. Dieperink and T. de Forest, Jr., Phys. Rev. C 10, 533 (1974)

[20] B. A. Brown, Phys. Rev. C 58, 220 (1998).

[21] B. A. Brown, W. A. Richter, and R. Lindsay, Phys. Lett. B 483, 49 (2000).

[22] B. A. Brown, S. Typel, and W. A. Richter, Phys. Rev. C 65, 014612 (2001).

[23] W. A. Richter and B. A. Brown, Phys. Rev. C 67, 034317 (2003).

[24] S. Typel and G. Baur, Phys. Rev. C 50, 2104 (1994).

[25] H. de Vries, C. V. de Jager, and C. de Vries, At. Data Nucl. Data Tables 36, 495 (1987).

[26] A. Ozawa, T. Suzuki, and I. Tanihata, Nucl. Phys. A693, 32 (2001).

[27] F. Ajzenberg-Selove, Nucl. Phys. A523, 1 (1991).

[28] K. Hencken, G. Bertsch, and H. Esbensen, Phys. Rev. C 54, 3043 (1996). 